\title{
Ultrafast VHE Gamma-Ray Flares of IC 310
}

\author{
Maxim V. Barkov ${ }^{1,2}$, Felix Aharonian ${ }^{3,4}$ and Dmitriy V. Khangulyan ${ }^{5}$ \\ ${ }^{1}$ DESY, Platanenallee 6, 15738 Zeuthen, Germany \\ email: maxim.barkov@desy.de \\ ${ }^{2}$ Astrophysical Big Bang Laboratory, RIKEN, 2-1 Hirosawa, Wako, Saitama 351-0198, Japan \\ email: maxim.barkov@riken.jp \\ ${ }^{3}$ Dublin Institute for Advanced Studies, 31 Fitzwilliam Place, Dublin 2, Ireland \\ ${ }^{4}$ Max-Planck-Institut für Kernphysik, Saupfercheckweg 1, D-69117 Heidelberg, Germany \\ ${ }^{5}$ Department of Physics, Rikkyo University, Nishi-Ikebukuro 3-34-1, Toshima-ku, Tokyo \\ 171-8501, Japan
}

\begin{abstract}
In 2012 November MAGIC detected a bright flare from IC 310. The flare consisted of two sharp peaks with a typical duration of $\sim 5 \mathrm{~min}$. The energy released during that event has been estimated to be at the level of $2 \times 10^{44} \mathrm{erg} \mathrm{s}^{-1}$.

In this work we derive an upper limit on the possible luminosity of flares generated in black hole $(\mathrm{BH})$ magnetosphere, which depends very weakly on the mass of $\mathrm{BH}$ and is determined by disk magnetisation, viewing angle, and pair multiplicity. Since all these parameters are smaller than a unit, the luminosity $2 \times 10^{43} \mathrm{erg} \mathrm{s}^{-1}$ can be taken as a strict upper limit for flare luminosity for several minutes variability time. This upper limit appears to be approximately an order of magnitude below the value measured with MAGIC. Thus, we conclude that it seems very unfeasible that the magnetospheric processes can be indeed behind the bright flaring activity recorded from IC 310 .
\end{abstract}

Keywords. gamma rays: theory, galaxies: active, galaxies: active

\section{Introduction}

The gamma-ray emission of blazars is strongly variable with fluxes which match well the sensitivity of the Fermi/LAT in the $\mathrm{MeV} / \mathrm{GeV}$ band and the current arrays of imaging atmospheric Cherenkov telescopes (IACT) like H.E.S.S., MAGIC, and Veritas in the VHE band. During the strongest flares of BL Lac objects like Mkn 421, Mkn 501 and PKS 2155-304 the energy fluxes of VHE gamma-rays can exceed $f_{\mathrm{VHE}}=10^{-10} \mathrm{erg} / \mathrm{cm}^{2} \mathrm{~s}$. Such fluxes can be studied with IACT arrays which provide huge, as large as $10^{9} \mathrm{~cm}^{2}$, detection areas, in almost background free regime with a detection rate exceeding $1 \mathrm{~Hz}$. This allows variability studies on timescales of minutes. The fluxes of flares in $\mathrm{MeV} / \mathrm{GeV}$ gamma-rays from powerful quasars like 3C 279 and 3C 454.3 can be significantly larger, $f_{\mathrm{VHE}}=10^{-8} \mathrm{erg} / \mathrm{cm}^{2} \mathrm{~s}$. But because of the limited detection area of Fermi/LAT and AGILE $\left(\simeq 1 \mathrm{~m}^{2}\right)$, the variability studies can be performed on hour timescales. It is interesting to compare these timescales with the minimum time that characterises a black hole system as an emitter, namely, the light crossing time of the gravitational radius of the black hole:

$$
\tau_{0}=r_{\mathrm{g}} / c=5 \times 10^{3} M_{9} \mathrm{~s} .
$$

Note that $r_{\mathrm{g}}=G M / c^{2}=1.5 \times 10^{14} M_{9} \mathrm{~cm}$ is the minimum value for the gravitational radius corresponding to the extreme Kerr black hole (i.e., twice smaller than the Schwarschild radius).

Thus, for a typical mass range of black holes in powerful AGN, $M \geqslant 10^{8} M_{\odot}$, the IACT arrays have a unique potential for exploring the physics of black holes close to 
the event horizon on timescales significantly shorter than $\tau_{0}$. Remarkably, such ultra-fast VHE gamma-ray flares events have been already detected from at least three AGN $\dagger, \mathrm{PKS}$ 2155-304 (Aharonian et al. 2007), Mkn 501 (Albert et al. 2007), and IC 310 (Aleksić et al. 2014). The GeV gamma-ray flares detected on timescales of minutes from $3 \mathrm{C} 279$ (Ackermann et al. 2016) and hours from 3C 279 (Hayashida et al. 2015) and 3C 454.3 (Abdo et al. 2011), are close to $\tau_{0}$ (the black hole masses in these objects are estimated in the intervals $(0.3-0.8) \times M_{9}$ (Woo \& Urry 2002; Gu et al. 2001; Nilsson et al. 2009) and $(0.5-4) \times M_{9}$ (Gu et al. 2001; Bonnoli et al. 2011), respectively). For comparison, the characteristic timescales of the even shortest ( $\sim 1 \mathrm{~ms}$; Pozanenko \& Loznikov 2002; Golkhou et al. 2015) gamma-ray burst events which associate, most likely, with solar mass black holes, exceed $\tau_{0}$ by several orders of magnitude.

The detection of the variable VHE gamma-ray emission from AGN on timescales significantly shorter than $\tau_{0}$ is an extraordinary result and requires a careful treatment and interpretation. If the emission is produced in a relativistically moving source the variability time-scale seen by an observer is shorten by the Doppler factor $\delta_{\mathrm{em}}=$ $1 / \Gamma_{\mathrm{em}}\left(1-\beta \cos \theta_{\mathrm{em}}\right)$, where $\Gamma_{\mathrm{em}}=1 / \sqrt{\left(1-\beta_{\mathrm{em}}^{2}\right)}$ and $\theta_{\mathrm{em}}$ are bulk Lorentz factor of the emitting source and the angle between its velocity and the line of sight, respectively. Thus if we want to increase the proper size of the emitter, $R^{\prime}$, (i.e. the source size in co-moving reference frame) to a reasonable value of $R^{\prime} \geqslant r_{\mathrm{g}}$, the Doppler factor should exceed, for example, in the case $\ddagger$ of PKS 2155-304, $\delta_{\mathrm{em}}=25$.

However, there is another issue of conceptual importance which cannot be ignored. The problem is that if the jet perturbations propagate from the central engine, e.g., in the form of sequence of blobs ejected with different Lorentz factors (leading to internal shocks), the size of the emitter in the laboratory frame does not depend on the Doppler factor. Let us define the proper size of the production region as $R^{\prime}=\lambda \Gamma_{\mathrm{j}} r_{\mathrm{g}}$, where $\Gamma_{\mathrm{j}}$ and $\lambda$ are the jet bulk Lorentz factor and a dimensionless parameter defining the size of the production region that should exceed $1(\lambda \geqslant 1)$ for the conventional assumptions. Combining the causality requirement and the limitation on the variability timescale, we have

$$
t_{\mathrm{var}} \geqslant \tau_{0} \frac{\lambda \Gamma_{\mathrm{j}}}{\Gamma_{\mathrm{em}}}
$$

The observational requirement of $t_{\mathrm{var}}=0.04 \tau_{0}$ inferred from the VHE flares of PKS 2155-304 (Aharonian et al. 2007) requires $\Gamma_{\mathrm{em}} \simeq 25 \lambda \Gamma_{\mathrm{j}}$, i.e., the emitter moves relativistically in the frame of the jet which, in its turn, moves relativistically towards the observer. The "jet in the jet" model suggested by Giannios et al. (2009) can be consider as a possible realization of this general scenario. Alternatively, i.e., if the source of the flare does not move relativistically in respect to the jet $\left(\Gamma_{\mathrm{em}} \simeq \Gamma_{\mathrm{j}}\right)$, the size of the source should be much less than the black hole's gravitational radius: $\lambda \simeq 0.04$. There are at least two reasons to invalidate the requirement of $\lambda>1$ : (1) the flare might originate in a small fraction of the BH magnetosphere, or (2) the perturbation that leads to the flare might have an external origin, i.e. not directly related to the black hole. An analogy for the first scenario could be emission of radio loud pulsars. In these objects the radio pulses are believed to originate in the polar cap region that substitute only a small fraction of the pulsar surface. Note that for the typical pulsar radius $R_{\mathrm{psr}}$ of $10 \mathrm{~km}$, $\tau_{0}=R_{\mathrm{psr}} / c \sim 30 \mu \mathrm{s}$ is too small to be probed through the variability of radio emission.

$\dagger$ For a recent summary of such systems see Vovk \& Babić (2015)

$\ddagger$ This estimate differs by a factor of 2 from the one derive in Aharonian et al. (2007) since in that paper one used the Schwarzschild radius for the estimate while we use the Kerr radius. 
The second possibility can be realized if a star or a cloud penetrates to the jet from outside (Barkov et al. 2010, 2012a,b; Bosch-Ramon et al. 2012; Khangulyan et al. 2013). Since the star radius $R_{*} \ll r_{\mathrm{g}}$, this scenario may initiate perturbations on scales smaller than the black hole's gravitation radius $r_{\mathrm{g}}$ (Barkov et al. 2012a).

Note that while all these three scenarios can explain, in principle, the detected time structures on scales $t_{\mathrm{var}}<\tau_{0}$, there are important additional requirements that should be fulfilled: (1) the overall energy budget should be feasible, (2) the emitter should be optically thin, i.e. gamma-rays should be able to escape their production region without significant absorption, (3) the proposed radiation mechanism should be able to explain the detected spectral features of gamma-radiation.

In this paper we discuss the fisibility of the source of the flare which could appear in a magnetospheric gap occupying a small volume in the proximity of the black hole close to the event horizon (Neronov \& Aharonian 2007; Levinson \& Rieger 2011);

\section{Addressing the "sub-horizon" scale variability}

Several groups argued that gamma-ray flares can be generated immediately in the magnetosphere of the central BH (see, e.g., Levinson \& Rieger 2011). However, in certain cases one does not validate feasibility of such a scenario from the point of the available power (see, e.g., Aleksić et al. 2014) or the minimum variability time scale (see, e.g., Hirotani \& $\mathrm{Pu} 2015$ ) . While in the case of flare production in relativistically moving blobs the apparent luminosity of the emission is strongly enhanced by the Doppler boosting and beaming effects, the emission generated in the magnetosphere allows to evaluate directly the true power spent for the flare production. Moreover, since the gap should collapse from both sides (Timokhin 2010), the observed variability time scale provided an upper limit on the size of the gap: $h<t_{\mathrm{var}} c$. As it is shown below, these two simple facts make possible a conclusion that the magnetospheric origin for the gamma-ray emission meets energy limitations that clearly exclude production of bright flares in the vicinity of the $\mathrm{BH}$. Importantly, the derived upper limit depends only marginally on the central $\mathrm{BH}$ mass, thus detection of such events possible only from the nearby supermassive BH, e.g. from M87 as suggested by Levinson \& Rieger (2011) or even from the Galactic center (we note here that due to unfavorable orientation of the line of sight the emission from the Galactic center may be severely attenuated). The later constraint has been ignored by Hirotani \& $\mathrm{Pu}$ (2015) for explanation of a very short flare detected with MAGIC from IC 310 (see, e.g., Aleksić et al. 2014).

The electrical field in the gap can be estimated as

$$
E \sim B_{\mathrm{bh}} \frac{R \Omega_{\mathrm{F}} \sin \theta}{c},
$$

where $B_{\mathrm{bh}}$ is the magnetic field at the $\mathrm{BH}$ horizon, $\Omega_{\mathrm{F}}$ is the angular velocity of the frame, $R$ is radius, and $\theta$ is polar angle. The gap potential drop can be estimated as

$$
\Delta V \lesssim h B_{\mathrm{bh}} \frac{R \Omega_{\mathrm{F}} \sin \theta}{c}
$$

where $h \sim t_{\mathrm{var}} c<r_{\mathrm{g}}$ is the thickness of the gap. An estimated difference by a factor $h / R$ from Eq. 2.2 is often used for the gap potential (see, e.g., Blandford \& Znajek 1977; Levinson \& Rieger 2011). The nature of the difference between these two estimates is related to influence of magnetospheric charges located outside the gap. The estimate given by Eq. 2.2 does not account for this contribution, and since the external charges, even if they are located outside the gap, tend to decrease the electrical field in the gap, Eq. 2.2 
provides a strict upper limit on the gap potential (see also Broderick \& Tchekhovskoy 2015).

The luminosity of the particles accelerated in the gap can be estimated as (Levinson \& Rieger 2011)

$$
L_{\gamma}<4 \pi R^{2} c \kappa \rho_{\mathrm{GJ}} \Delta V
$$

where $\kappa$ and $\rho_{\mathrm{GJ}}=\Omega_{\mathrm{F}} B_{\mathrm{bh}} \sin \theta /(2 \pi c)$ are pair multiplicity and Goldreich-Julian charge density, respectively. For an efficient operation of the gap acceleration an obvious condition $\kappa \ll 1$ should be fulfilled.

For a Kerr BH with maximum angular momentum the angular velocity $\Omega_{\mathrm{F}}$ can be estimated as:

$$
\frac{\Omega_{\mathrm{F}}}{c} \simeq \frac{1}{4 r_{g}} .
$$

Thus, taking $R=r_{\mathrm{g}}$ (otherwise, one can account for the drop of the magnetic field strength away from the horizon) one obtains

$$
L_{\gamma}<\frac{1}{8} \kappa B_{\mathrm{bh}}^{2} r_{\mathrm{g}} h c \sin ^{2} \theta
$$

We should note that if $h \rightarrow r_{\mathrm{g}}$ the luminosity estimate provided by Eq. (2.5) (after averaging over polar angle $\theta$ ) exceeds by a factor of 2 the luminosity of the BlandfordZnajek mechanism (Blandford \& Znajek 1977). This indicates that Eq. (2.5) can be taken as a safe upper limit for the available power.

For a minute variability time-scale, $t_{\mathrm{var}}=5 t_{\mathrm{var}, 5}$ min, the gap thickness $h=10^{13} t_{\mathrm{var}, 5} \mathrm{~cm}$ is small as compared to the gravitational radius of supermassive $\mathrm{BH}$ with $M_{8}>1$, the estimated gamma-ray luminosity cannot exceed the following value:

$$
L_{\gamma}<5 \times 10^{43} \kappa B_{4}^{2} M_{8} t_{\mathrm{var}, 5} \sin ^{2} \theta \mathrm{erg} \mathrm{s}^{-1},
$$

where $B_{\mathrm{bh}}=10^{4} B_{4} \mathrm{G}$.

Equation (2.6) contains two parameters that are determined by the condition in the close vicinity of the $\mathrm{BH}$ : pair multiplicity, $\kappa$, and the magnetic field strength, $B$. Importantly, these parameters are essentially defined by the same process: by the accretion rate. Magnetic field at the $\mathrm{BH}$ horizon needs to be supported by accretion disk, therefore the field strength is directly determined by the accretion rate. On the other hand, the accretion rate also defines the intensity of photon fields in the magnetosphere, and consequently the multiplicity parameter (see, e.g., Levinson \& Rieger 2011). The multiplicity parameter should remain strictly below unity, since otherwise the gap potential will be completely screened, this implies an upper limit on the accretion rate, and consequently on the magnetic field strength.

In previous works by Levinson \& Rieger (2011); Aleksić et al. (2014) the estimated value of the maximum accretion rate compatible with existence of a vacuum gap in magnetosphere was

$$
\dot{m}<3 \times 10^{-4} M_{8}^{-1 / 7},
$$

where $\dot{m}$ is accretion rate measured in the Eddington units:

$$
\dot{M}_{\mathrm{edd}}=\frac{4 \pi m_{p} G M_{\mathrm{BH}}}{\eta c \sigma_{t}} .
$$

Here $m_{p}$ and $\sigma_{t}$ are mass of proton and Thompson cross-section, respectively. Efficiency of the accretion, $\eta$, determines a fraction of gravitational energy that is radiated by the accretion flow. To derive the estimate provided by Eq. (2.7), Levinson \& Rieger (2011) adopted a value of $\eta=0.1$. Levinson \& Rieger (2011) also have provided an estimate for 
magnetic field strength at the $\mathrm{BH}$ horizon as

$$
B_{\mathrm{bh}}=1.3 \times 10^{5}\left(\dot{m} / M_{8}\right)^{1 / 2} \mathrm{G},
$$

where we rescaled the numerical coefficient to the normalization used through out our paper. Therefore, one obtains an estimate on the maximum luminosity achievable at magnetospheric gap as

$$
L_{\gamma}<3 \times 10^{42} \kappa M_{8}^{-1 / 7} t_{\mathrm{var}, 5} \sin ^{2} \theta \operatorname{erg~s}^{-1} .
$$

The numerical coefficient in the above estimate differs (100 times smaller) from the value obtained by Levinson \& Rieger (2011), which is related to the fact that we used Kerr radius while Levinson \& Rieger (2011) provided the estimate for the Schwardschild radius. Such an approach is justified in the case of M87 given the relatively low gammaray flux and large overall uncertainties in this estimate. In other cases, e.g., for IC 310, the energy budget is much tighter, therefore below we provide a somewhat more accurate treatment of the case of magnetosphere around a Kerr BH. For example, the accretion efficiency of a flow accreted by a Kerr BH should be rather high $\eta=0.3$ (see, e.g., Thorne 1974). Theoretically predicted value is, in fact, higher, $\eta=0.42$ (Bardeen et al. 1972), but gravitational redshift and photon aberration should decrease it.

The strength of the magnetic field can be obtained by extrapolating the conditions at the last marginally stable orbit. Let us assume that the magnetic field in the disk is:

$$
B_{\mathrm{d}}=\sqrt{8 \pi \beta_{\mathrm{m}} p_{g}}
$$

here $\beta_{\mathrm{m}}$ and $p_{g}$ are disk magnetization and gas pressure in accretion disk that supports the magnetic field near the horizon. The gas pressure can be estimated using the solution for radiatively inefficient accretion flow (see Narayan \& Yi 1994) (more accurate treatment of accretion flow revile correction less than 20-30\% (see Narayan \& Yi 1995)) as

$$
p_{g}=\frac{\sqrt{10} \dot{M} \sqrt{G M_{\mathrm{BH}}}}{12 \pi \alpha_{s s} R^{5 / 2}},
$$

here $\alpha_{s s}$ is non-dimensional viscosity parameter in the disk (Shakura \& Sunyaev 1973). This reduces Eq. (2.11) to the following form:

$$
B_{\mathrm{bh}}=1.5 \frac{\beta_{\mathrm{m}}^{1 / 2}(\dot{M} c)^{1 / 2}}{\left(\alpha_{s s}\right)^{1 / 2} r_{\mathrm{g}}} .
$$

Then the total gamma-ray luminosity, Eq. (2.5), can be estimated as

$$
L_{\gamma}<\frac{\sqrt{10}}{12} \frac{\beta_{\mathrm{m}} \kappa\left(h / r_{g}\right) \sin ^{2} \theta \dot{M} c^{2}}{\alpha_{s s}} .
$$

For the sake of consistency we will estimate the multiplicity coefficient, $\kappa$, at Kerr radius through the density of the photons in the magnetosphere (see, e.g., Levinson \& Rieger 2011) as

$$
\kappa \equiv \frac{n_{ \pm}}{n_{\mathrm{GJ}}} \approx 6 \times 10^{6} \frac{\dot{m}^{7 / 2} M_{\mathrm{BH}, 8}^{1 / 2}}{\left(\eta \alpha_{s s}\right)^{7 / 2} \beta_{\mathrm{m}}^{1 / 2}} .
$$

Thus, one obtains an upper limit on the accretion rate allowing magnetospheric emission as:

$$
\dot{m}<10^{-2} \frac{\eta \alpha_{s s} \beta_{\mathrm{m}}^{1 / 7}}{M_{\mathrm{BH}}^{1 / 7}} .
$$


Equations (2.16) and (2.8) substituted to Eqs. (2.13) and (2.14) yield estimates for the largest magnetic field that is consistent with vacuum gap

$$
B_{\mathrm{bh}}<5 \times 10^{3}\left(\frac{\beta_{\mathrm{m}}}{M_{\mathrm{BH}, 8}}\right)^{4 / 7} \mathrm{G}
$$

and the maximum luminosity of particles accelerated by the gap potential drop

$$
L_{\gamma}<2 \times 10^{43} \beta_{\mathrm{m}}^{8 / 7} \kappa t_{v a r, 5} M_{\mathrm{BH}, 8}^{-1 / 7} \sin ^{2} \theta \text { erg s}{ }^{-1} .
$$

This limit is independent on $\alpha_{s s}$ and $\eta$. We can see that the derived upper limit on photon luminosity is significantly smaller than the observed VHE luminosity of $2 \times 10^{44} \mathrm{erg} \mathrm{s}^{-1} \dagger$. In addition, several factors can reduce the available power even further: (i) for favorable viewing angles, the luminosity may be damped by a large factor $\sin ^{2} \theta<0.1$; (ii) photonphoton absorption on local infrared field may be severe in powerful AGNs.

Given the weak dependence of Eq. (2.18) on the BH mass, and possible strong impact of the gamma-gamma absorption in case of large masses of BHs, detection of magnetospheric short flares from nearby lighter $\mathrm{BH}$ viewed by angle $\lesssim \pi / 4$ (to avoid severe absorption in the accretion disk) should be more feasible.

\section{Discussion and Conclusions}

Equation (2.18) determines the maximum luminosity of vacuum gaps that can collapse quicker than $t_{\mathrm{var}}$. It has been assumed for its derivation that magnetic field is determined by accretion regime that in turn determines the intensity of the photon field in the magnetosphere. In the case of the steady accretion, this seems to be a very feasible approximation, however this may look less certain in the case of rapidly changing accretion rate, since the processes that govern change in the accretion rate and escape of the magnetic field may have different characteristic timescales. The dominant contribution to the photon field is coming from plasma located at distances $r \sim 2 r_{g}$, and the characteristic viscous accretion time (density decay time in the disk) is

$$
t_{\rho, \text { decay }} \simeq \frac{2 r_{\mathrm{g}}}{c \alpha_{\mathrm{ss}}} \simeq 10^{4} \alpha_{\mathrm{ss},-1}^{-1} M_{8} \mathrm{~s} .
$$

When the accretion fades, the decay of the magnetic field is determined by magnetic field reconnection rate (Komissarov 2004), i.e.

$$
t_{\mathrm{B}, \text { decay }} \simeq \frac{\pi r_{\mathrm{g}}}{0.3 c} \sim 10^{4} M_{8} \mathrm{~s} .
$$

Since these two time-scales are essentially identical, it is natural to expect that the field strength and disk density will decay simultaneously. Thus, Equation (2.18) should also be valid for time-variable accretion regime.

According to the estimate provided by Eq. (2.18) the possible luminosity of flares generated in $\mathrm{BH}$ magnetosphere depends very weakly on the mass of $\mathrm{BH}$ and is determined by disk magnetization, viewing angle, and pair multiplicitył. Since all these parameters are smaller than unit, then the numerical coefficient in Eq. (2.18) can be taken as a strict

$\dagger$ In the paper (Hirotani \& Pu 2015) the efficiency of BZ mechanism was required on the level $10000 \%$, unfortunately in 3D simulations McKinney et al. (2012) the efficiency is limited by value $300 \%$. We should remark that equation (2.14) with $\alpha_{\mathrm{ss}}=0.1$ gets efficiency on the level $250 \%$ that is very close to results of $3 \mathrm{D}$ simulations.

$\ddagger$ Equation (2.18) does not account for relativistic effects that should be small unless the gap is formed close to the horizon. If the vacuum gap is close to the horizon, then gravitational redshift should even strengthen the constraints imposed by the variability time. 
upper limit for flare luminosity for the given variability time. This upper limit appears to be approximately an order of magnitude below the value measured with MAGIC (Aleksić et al. 2014). Thus, we conclude that it seems very unfeasible that this processes can be indeed behind the bright flaring activity recorded from IC 310.

\section{References}

Abdo, A. A., et al. 2011, ApJ, 733, L26

Ackermann, M., et al. 2016, ApJ, 824, L20

Aharonian, F., et al. 2007, ApJ, 664, L71

Albert, J., et al. 2007, The Astrophysical Journal, 669, 862

Aleksić, J., et al. 2014, Science, 346, 1080

Bardeen, J. M., Press, W. H., \& Teukolsky, S. A. 1972, ApJ, 178, 347-370

Barkov, M. V., Aharonian, F. A., Bogovalov, S. V., Kelner, S. R., \& Khangulyan, D. 2012a, ApJ, 749, 119

Barkov, M. V., Aharonian, F. A., \& Bosch-Ramon, V. 2010, ApJ, 724, 1517

Barkov, M. V., Bosch-Ramon, V., \& Aharonian, F. A. 2012b, ApJ, 755, 170

Blandford, R. D., \& Znajek, R. L. 1977, MNRAS, 179, 433

Bonnoli, G., Ghisellini, G., Foschini, L., Tavecchio, F., \& Ghirlanda, G. 2011, MNRAS, 410, 368

Bosch-Ramon, V., Perucho, M., \& Barkov, M. V. 2012, A $\xi A$, 539, A69

Broderick, A. E., \& Tchekhovskoy, A. 2015, ArXiv e-prints

Giannios, D., Uzdensky, D. A., \& Begelman, M. C. 2009, MNRAS, 395, L29

Golkhou, V. Z., Butler, N. R., \& Littlejohns, O. M. 2015, ArXiv e-prints

Gu, M., Cao, X., \& Jiang, D. R. 2001, MNRAS, 327, 1111

Hayashida, M., et al. 2015, ApJ, 807, 79

Hirotani, K., \& Pu, H.-Y. 2015, ArXiv e-prints

Khangulyan, D. V., Barkov, M. V., Bosch-Ramon, V., Aharonian, F. A., \& Dorodnitsyn, A. V. 2013, ApJ, 774, 113

Komissarov, S. S. 2004, MNRAS, 350, 427

Levinson, A. \& Rieger, F. 2011, ApJ, 730, 123

McKinney, J. C., Tchekhovskoy, A., \& Blandford, R. D. 2012, MNRAS, 423, 3083

Narayan, R. \& Yi, I. 1994, ApJ, 428, L13

-. 1995, ApJ, 444, 231-243

Neronov, A. \& Aharonian, F. A. 2007, ApJ, 671, 85

Nilsson, K., Pursimo, T., Villforth, C., Lindfors, E., \& Takalo, L. O. 2009, A $\mathscr{G} A$, 505, 601

Pozanenko, A., \& Loznikov, V. 2002, in Lighthouses of the Universe: The Most Luminous Celestial Objects and Their Use for Cosmology, ed. M. Gilfanov, R. Sunyeav, \& E. Churazov, 194

Shakura, N. I. \& Sunyaev, R. A. 1973, A\&A, 24, 337

Thorne, K. S. 1974, ApJ, 191, 507

Timokhin, A. N. 2010, MNRAS, 408, 2092

Vovk, I. \& Babić, A. 2015, A\&A, 578, A92

Woo, J.-H. \& Urry, C. M. 2002, ApJ, 579, 530 